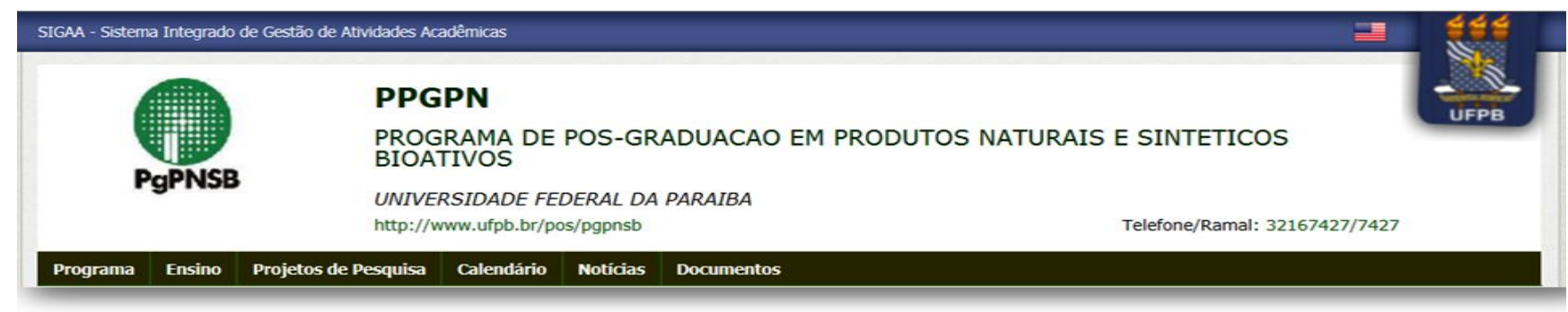

\title{
SciForum
}

MOL2NET

\section{Molecular docking studies of benzothiazinone derivatives in the search for new tuberculostatic agents}

Jéssika de Oliveira Viana (viana_jess@ hotmail.com) ${ }^{1}$, Marcus Tullius Scotti (mtscotti@gmail.com) ${ }^{1}$, Luciana Scotti (luciana.scotti@gmail.com) ${ }^{1}{ }^{1}$.

${ }^{1}$ 1Program of Natural and Synthetic Bioactive Products (PgPNSB), Health Sciences Center, Federal University of Paraíba,, João Pessoa-PB, Brazil

* Correspondence: luciana.scotti@gmail.com; Tel.: +55-83-3291-1528

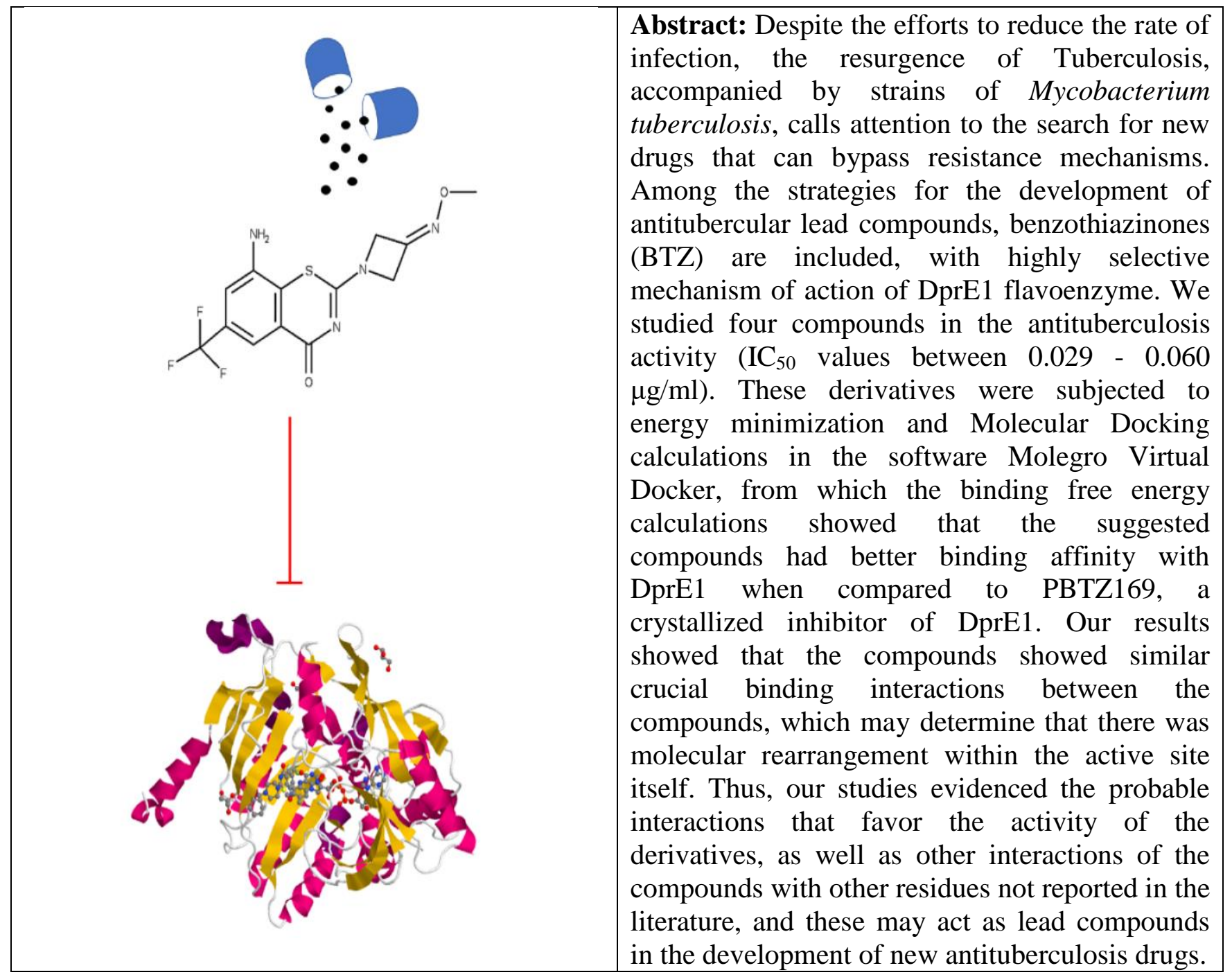

Keywords: Tuberculosis, benzothiazinones, Docking 


\section{Introduction}

The Tuberculosis (TB) is a contagious bacterial infection caused by a group of bacteria known as Mycobacterium, which comprises the bacillus Mycobacterium tuberculosis (Mtb). This is an optional intracellular pathogen that has developed resistance to first- and second-line anti-tuberculosis drugs. Antibiotic resistance and multidrug resistant TB strain are a serious problem because of the negative effects on the design of treatment strategies. Therefore, new classes of anti-mycobacterial compounds are required [1].

In recent years, a number of significant efforts have been made worldwide to treat tuberculosis [2]. Despite all current efforts in the development of anti-tuberculosis drugs, the fact is that only two new anti-TB drugs have been approved after a period of approximately 40 years. In addition, these drugs are only recommended for MDR-TB (multi-drug resistant $\mathrm{TB}$ ) while other treatment options are not available [3-4].

The 1,3-benzothiazin-4-ones, also known as benzothiazinones (BTZ), are a new class of potent antimycobacterial agents responsible for blocking the synthesis of D-Arabinofuranose, a component of arabinogalactan and arabinomannan, with a mechanism of action highly selective in the cell wall of mycobacteria [5]. BTZ043, as well as its derivative PBTZ169, is a nitroaromatic compound that acts on the subunit of the enzyme DprE1, an essential flavoprotein for the cell membrane of Micobacterium tuberculosis [6-8]. BTZ043, which contains activity similar to clinical isolates for M. tuberculosis [6], showed nanomolar activity [5], making it a promising candidate against tuberculosis.

Therefore, in this work we analyzed four benzothiazinones derivatives recently published by Zhang et al. (2017), which have already undergone synthesis, elucidation and in vitro tests and have shown promising activity against Mycobacterium tuberculosis. Soon after, we evaluated its inhibition in the DprE1 enzyme of the derivatives through Molecular Docking.

We also observed that some of the 4benzothiazinones and PBTZ169 derivatives with the receptor amino acid residues were similar, highlighting them as critical interactions for the activity: ketone oxygen with residue Lys 134, steric interaction of Fluorine with Lys367, and the different portions of each molecule interacted with the Cys 187 residue, which may determine that there was molecular rearrangement within the active site itself (Fig. 1) and explain how these residues are essential for the activity.

Table 1. Energy Values of ligand and enzyme DprE1 interaction calculated from Molegro Virtual Docker program.

\begin{tabular}{c|c}
\hline Compound & MolDock score \\
\hline PBTZ169 & -70.0994 \\
\hline $10 \mathrm{a}$ & -72.5120 \\
\hline $10 \mathrm{e}$ & -59.0168 \\
\hline $10 \mathrm{~h}$ & -64.1054 \\
\hline $11 \mathrm{a}$ & -58.0330 \\
\hline
\end{tabular}




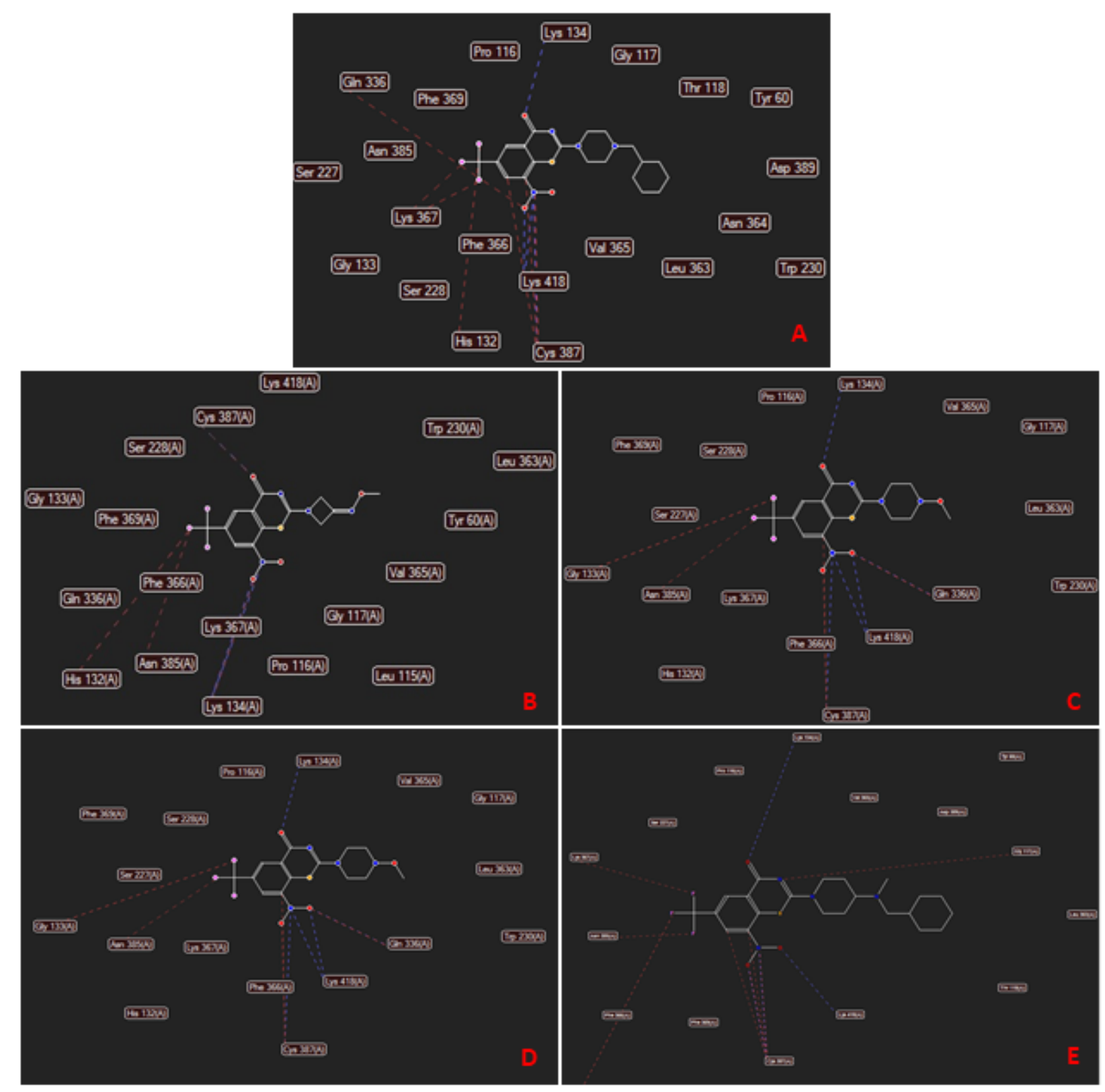

Figure 1. Hydrogen and steric bonds between the compounds and DprE1. a) PBTZ169, b) 10A, c) 10E, d) 10H and e) $11 \mathrm{~A}$.

For compound 10a steric interactions of Fluor with Asn385 and His132 were observed; hydrogen bonds at the residues of Cys387, Lys134 and Lys367 through the carbonyl portion of the heterocyclic and the portion attached to the nitro portion of the benzene ring. For compounds 10e hydrogen bonds were also observed at the residues Cys387 and Lys418 with the nitrobenzene group, the residue Lys418 with the oxygen bound to nitrobenzene and hydrogen interactions of the residue Lys134 with the oxygen of the carbonyl of the heterocyclic.
The steric interactions were observed at residues Gly133 and Asn385 on fluorine bound to the benzene ring, at the residue Cys387 to benzene and at Gln336 at the oxygen of nitrobenzene.

For compound $10 \mathrm{~h}$, hydrogen bonds were observed at residues Lys418 and Cys387 to nitrobenzene and from Lys 134 the carbonyl portion of the heterocyclic. Steric interactions were observed at the residues of Lys387 with the trifluoromethylbenzene moiety, from Cys387 with nitrobenzene oxygen and directly with the benzene ring and from Gln338 with nitrobenzene oxygen. 
As a final analysis, for compounds 11a hydrogen interactions were observed in the Lys418 residues with the oxygen of the nitrobenzene moiety; the second interaction of the Cys387 residue with the nitrogen and oxygen of the nitrobenzene moiety and also the residue Lys134 with the oxygen of the carbonyl moiety of the heterocyclic. Steric interactions were observed at the Gly133, Asn385 and Lys367 residues with the trifluoromethylbenzene moiety, interactions of the two-carbon Cys387s with respect to the benzene group and interactions of the Gly117 residue with the nitrogen of the heterocyclic moiety.

Recently, studies by Shaikh et al. (2016) has shown that its benzothiazinone derivatives have similar binding modes reported in this study, from which the hydrogen bonding acts as a support and guides the position of the compound in the active site, favoring the other steric interactions with the receptor. In addition, the interactions of the benzene ring, correlating the pi-pi interaction, favored the activity of compound 11a in the active site of DprE1.

\section{Materials and Methods}

The three-dimensional structures were drawn using HyperChem 8.0.3 software [9] and energyminimized employing the $\mathrm{MM}+$ force field. Posteriorly, we performed a new geometry optimization based on the semi-empirical AM1 method on the Spartan program [10]. The optimized structures were subjected to conformational analysis using a random search method.

The four benzothiazinones and the PBTZ169 ligands were submitted to docking with enzyme DprE1 (ID PDB 4NCR) [11]. The enzymes were imported from the Protein Data Bank (PDB) in the Molegro Virtual Docker (MVD) 6.0 program. The Moldock score algorithm was used as a score function in predicting the best interaction between ligand and receptor.

\section{Conclusions}

In this study were analyzed by means of Molecular docking 4 new benzothiazinone derivatives in the enzyme DprE1, of which they had better energy values when compared with the standard compound. As well, these compounds obtained stability of binding to the amino acid residues of the active site. Therefore, the study emerged as an aid in the continuous search for new drug candidates that may have greater antituberculose activity in the enzyme DprE1, blocking the mycobacterial formation.

\section{References}

1. Herrera, J.L.; Costa, M.C.; Gonzalez, H.G.; Rodrigues, A.I.; Castilho, P.C. Synergistic antimycobacterial activities of sesquiterpene lactones from Laurus spp. J. Antim. Chemother., 2007, 59(3), 548-552.

2. Ryan, N.J.; Lo, J.H. Delamanid: First Global Approval. Drugs, 2014, 74(9), 10411045.

3. World Health Organization. Global Tuberculosis Report 2017. http://www.who.int/campaigns/tbday/2017/en/ (Accessed September 17, 2017).

4. Mulu, W.; Mekonnen, D.; Yimer, M.; Admassu, A.; Abera, B. Risk factors for multidrug resistant tuberculosis patients in Amhara National Regional State. African Health Sci., 2015, 15(2), 368-377.

5. Makarov, V.; Manina, G.; Mikusova, K.; Möllmann, Y.; Ryabova, O.; Saint-Joanis, B.; Dhar, N.; Pasca, M.R.; Bobovska, A.; Dianiskova, P.; Kordulakova, J.; Sala, C.; Fullam, E.; Schneider, P.; Mckinney, J.D.; Brodin, P.; Christophe, T.; Waddell, S.; Butcher, P.; Albrethsen, J.; Rosenkrands, I.; Brosch, R.; Nandi, V.; Bharath, S.; Gaonkar, S.; Shandil, R.K.; Balasubramanian, V.; Balganesh, T.; Tyagi, S.; Grosset, J.; Riccardi, G.; Cole, S.T. Benzothiazinones kill Mycobacterium tuberculosis by 
blocking arabinan synthesis. Science, 2009, 324(5928), 801-804.

6. Manina, G.; Pasca, M.R.; Buroni, S.; Rossi, E.; Riccardi, G.; Decaprenylphosphoryl- $\beta$ D-Ribose 2'-Epimerase from Mycobacterium tuberculosis is a Magic Drug Target. Curr. Med. Chem., 2010, 17(27), 3099-3108.

7. Trefzer, C.; Gonzalez, M.R.; Hinner, M.J.; Schneider, P.; Makarov, V.; Cole, S.T.; Johnsson, K. Benzothiazinones: Prodrugs That Covalently Modify the Decaprenylphosphoryl- $\beta$-D-ribose 2'epimerase DprE1 of Mycobacterium tuberculosis. J. Am. Chem. Soc., 2010, 132, 13663-13665.

8. Neres, J.; Pojer, F.; Molteni, E.; Chiarelli, L.R.; Dhar, N.; Boy-Röttger, S.; Buroni, S.; Fullam, E.; Degiacomi, G.; Lucarelli, A.P.; Read, R.J.; Zanoni, G.; Edmondson, D.E.; Rossi, E.; Pasca, M.R.; Mckinney, J.D.; Dyson, P.J.; Riccardi, G.; Mattevi, A.; Cole,
S.T.; Binda, C. Structural Basis for Benzothiazinone-Mediated Killing of Mycobacterium tuberculosis. Sci. Transl. Med., 2012, 4(50), 121-150.

9. Zhang, Y. The magic bullets and tuberculosis drug targets. Annu. Rev. Pharmacol. Toxicol., 2005, 45, 529-564.

10. HyperChem, Version 8.0; Hybercube Inc.: Gainesville, FL, USA, 2009.

11. Spartan model homepage for windows, available at http://www.wavefun.com/products/windows /SpartanModel/win_model.html, access in 4/5/2015.

12. Shaikh, M.H.; Subhedar, D.D.; Shingate, B.B.; Khan, F.A.K.; Sangshetti, J.N.; Khedkar, V.M.; Nawale, L.; Sarkar, D.; Navale, G.R.; Shinde, S.S. Synthesis, biological evaluation and molecular docking of novel coumarin incorporated triazoles as antitubercular, antioxidant and antimicrobial agents. Med. Chem. Research, 2016, 25(4), 790-804. 